\title{
Bifurcation Analysis on the Mathematical Model of Measles Disease Dynamics
}

\author{
Samuel Abubakar*, Ninuola Ifeoluwa Akinwande., Sirajo Abdulrahman . Festus Abiodun Oguntolu \\ Department of Mathematics/Statistics,Federal University of Technology, Minna, Nigeria \\ *Corresponding Author: sam.abu@futminna.edu.ng
}

Copyright (C) 2013 Horizon Research Publishing All rights reserved.

\begin{abstract}
In this paper we proposed a Mathematical model of Measles disease dynamics. The Disease Free Equilibrium (DFE) state, Endemic Equilibrium (EE) states and the characteristic equation of the model were obtained. The condition for the stability of the Disease Free equilibrium state was obtained. We analyze the bifurcation of the Disease Free Equilibrium (DFE) and the result of the analysis was presented in a tabular form.
\end{abstract}

Keywords Equilibrium State, Characteristic Equation, Stability

\section{Introduction}

Bifurcation theorem is concerned with dynamical systems which contain one or more external parameters and with the manner in which the solution set may undergo structural changes as the parameters are varied. Such behaviour is essentially determined by the stability of solutions and the manner in which this may change as the parameters vary [2]. When modeling measles dynamics, or those of any other microparasitic disease, the number or density of individuals in each stage of the disease is the important quantity to keep track of whereas the viral load per person is relatively unimportant.[3]. Following the classical approach, the population is divided into a susceptible, an infectious (and infected), and a recovered class, denoted by $S, I$ and $R$, respectively.

Measles, also known as rubeola or morbilli, is an infection of the respiratory system caused by a virus, specifically a paramyxovirus of the genus Morbillivirus. Morbilliviruses, like other paramyxoviruses, are enveloped, single-stranded, negative-sense RNA viruses. Symptoms include fever, cough, runny nose, red eyes and a generalized, maculopapular, erythematous rash.

\section{Materials and Methods}

\subsection{The SIR Model}

$$
\begin{aligned}
& \frac{\mathrm{dS}}{\mathrm{dt}}=\beta N-\alpha S I-\mu \mathrm{S} \\
& \frac{d I}{d t}=\alpha S I-(\gamma+\delta+\mu) I \\
& \frac{d R}{d t}=\gamma I-\mu R
\end{aligned}
$$

Where

$\beta=$ Birth rate

$\alpha=$ contact rate

$\mu=$ Natural death rate

$\mathrm{S}=$ Susceptible

$\gamma=$ Recovery rate

$\mathrm{I}=$ Infected

$\delta=$ Death rate due to disease

$\mathrm{R}=$ Removed with immunity/ Recovery

$N=\mathrm{S}+\mathrm{I}+\mathrm{R}$

set, $N=1$, i.e. assuming a closed population for a given period of time.

\subsection{Equilibrium State of the Model}

$$
\begin{gathered}
\text { At equilibrium } \frac{d S}{d t}=\frac{d I}{d t}=\frac{d R}{d t}=0 \\
\text { Let } S=x, I=y \text { and } R=z \\
\beta-\alpha x y-\mu x=0 \\
\alpha x y-(\gamma+\delta+\mu) y=0 \\
\gamma y-\mu z=0
\end{gathered}
$$

\subsection{The Disease Free Equilibrium (DFE)}

The equilibrium state in the absence of infection is known as Disease Free Equilibrium (DFE) and is such that, $\mathrm{y}=0$, From (5) 


$$
[\alpha x-(\gamma+\delta+\mu)] y=0
$$

Either $y=0$ or $\alpha x-(\gamma+\delta+\mu)=0$ If $y=0$, then we have

$$
\begin{gathered}
x=\frac{\beta}{\mu} \\
\mathrm{y}=0 \text { and } \mathrm{z}=0
\end{gathered}
$$

Therefore the Disease Free equilibrium is:

$$
(x, y, z)=\left(\frac{\beta}{\mu}, 0,0\right)
$$

\subsection{The Endemic Equilibrium (EE) State}

The equilibrium state with the presence of infection (i. e. y $\neq 0)$ is known as endemic equilibrium or non- zero equilibrium.

if $y \neq 0$ then we have from (7)

$$
\begin{gathered}
\therefore \alpha-(\gamma+\delta+\mu)=0 \\
x=\frac{\gamma+\delta+\mu}{\alpha} \\
y=\frac{\alpha \beta-\mu(\gamma+\delta+\mu)}{\alpha(\gamma+\delta+\mu)} \\
z=\frac{\alpha \beta \gamma-\mu \gamma(\gamma+\delta+\mu)}{\alpha \mu(\gamma+\delta+\mu)}
\end{gathered}
$$

Then, the endemic equilibrium state is given as:

$(x, y, z)=\left(\frac{\gamma+\delta+\mu}{\alpha}, \frac{\alpha \beta-\mu(\gamma+\delta+\mu)}{\alpha(\gamma+\delta+\mu)}, \frac{\alpha \beta \gamma-\mu \gamma(\gamma+\delta+\mu)}{\alpha \mu(\gamma+\delta+\mu)}\right)$

\subsection{The Characteristic Equation}

The Jacobian Matrix of the system is given by:

$J=\left[\begin{array}{ccc}-(\alpha y+\mu) & \alpha x & 0 \\ \alpha y & \alpha x-(\gamma+\delta+\mu) & 0 \\ 0 & \gamma & -\mu\end{array}\right]$

Thus, the $\operatorname{det}|J-\lambda I|$ is

$\left|\begin{array}{ccc}-(\alpha y+\mu+\lambda) & \alpha x & 0 \\ \alpha y & \alpha x-(\gamma+\delta+\mu)-\lambda & 0 \\ 0 & \gamma & -(\mu+\lambda)\end{array}\right|=0$ i.e.

$$
\begin{array}{r}
(\alpha y+\mu+\lambda)[[\alpha x-(\gamma+\delta+\mu)-\lambda](\mu+\lambda)] \\
+\alpha^{2} x y(\mu+\lambda)=0
\end{array}
$$

Equation (15) is our characteristic equation But recall that the DFE is given as:

$$
(x, y, z)=\left(\frac{\beta}{\mu}, 0,0\right)
$$

Then,

$$
\operatorname{det}|J-\lambda I|=\left|\begin{array}{ccc}
-(\mu+\lambda) & \alpha \frac{\beta}{\mu} & 0 \\
0 & \alpha \frac{\beta}{\mu}-(\gamma+\delta+\mu)-\lambda & 0 \\
0 & \gamma & -(\mu+\lambda)
\end{array}\right|=0
$$

$(\mu+\lambda)^{2}\left[\alpha \frac{\beta}{\mu}-(\gamma+\delta+\mu)-\lambda\right]=0$

Either

$$
(\mu+\lambda)^{2}=0 \text { or } \alpha \frac{\beta}{\mu}-(\gamma+\delta+\mu)-\lambda=0
$$

Therefore, $\lambda_{1}=-\mu, \lambda_{2}=-\mu$, and $\mathrm{s}$

$$
\lambda_{3}=\alpha \frac{\beta}{\mu}-(\gamma+\delta+\mu)
$$

The condition for stability of DFE is that the eigen values $\lambda_{i}<0 ; \quad i=1,2,3$;

From (17)

$\lambda_{1}<0$ and $\lambda_{2}<0$

$\lambda_{3}<0$ if $\alpha \frac{\beta}{\mu}<(\gamma+\delta+\mu)$

$\lambda_{3}>0$ if $\alpha \frac{\beta}{\mu}>(\gamma+\delta+\mu)$

Hence, the DFE is stable if $\alpha \frac{\beta}{\mu}<(\gamma+\delta+\mu)$ and unstable if $\alpha \frac{\beta}{\mu}>(\gamma+\delta+\mu)$

\subsection{Bifurcation at Equilibrium State}

Recall from (17) $\lambda_{3}=\frac{\alpha \beta}{\mu}-(\gamma+\delta+\mu)$

The Disease Free Equilibrium state is stable if 


$$
\frac{\alpha \beta}{\mu}<(\gamma+\delta+\mu)
$$

We use the inequality above to obtain a bifurcation parameter from the Disease Free Equilibrium state

From (17b) we have:

$$
\beta<\frac{\mu}{\alpha}(\gamma+\delta+\mu)
$$

Define,

$$
\omega=\frac{\mu}{\alpha}(\gamma+\delta+\mu)-\beta
$$

Let $\omega$ be a bifurcation parameter from the Disease Free Equilibrium state, if $\omega<0$ the disease free equilibrium state will be stable and unstable if $\omega>0$

It follows that as $\omega$ transit from positive to negative there is likelihood of disease outbreak.

From (19)

$$
\mu^{2}+(\gamma+\delta) \mu-(\beta+\omega) \alpha=0
$$

Making $\mu$ the subject gives

$$
\mu=\frac{-(\gamma+\delta)+\sqrt{(\gamma+\delta)^{2}+4 \alpha(\beta+\omega)}}{2}
$$

or

$$
\mu=\frac{-(\gamma+\delta)-\sqrt{(\gamma+\delta)^{2}+4 \alpha(\beta+\omega)}}{2}
$$

Taking the positive value of $\mu$ Expanding the characteristic equation (15) we have:

$$
\begin{aligned}
& \lambda^{3}-[\alpha(x-y)-(\gamma+\delta+3 \mu)] \lambda^{2} \\
& -\left[\begin{array}{l}
\alpha \mu(2 x-y) \\
+2 \alpha^{2} x y \\
-(\gamma+\delta+\mu)(\alpha y+2 \mu) \\
-\mu^{2}
\end{array}\right] \lambda \\
& -2 \alpha^{2} \mu x y+(\gamma+\delta+\mu)\left(\alpha \mu y+\mu^{2}\right) \\
& -\alpha \mu^{2} x=0
\end{aligned}
$$

Setting

$$
\begin{gathered}
A=-[\alpha(x-y)-(\gamma+\delta+3 \mu)] \\
B=-\left[\alpha \mu(2 x-y)+2 \alpha^{2} x y-\right. \\
\left.(\gamma+\delta+\mu)(\alpha y+2 \mu)-\mu^{2}\right] \\
C=(\gamma+\delta+\mu)\left(\alpha \mu y+\mu^{2}\right)-2 \alpha^{2} \mu x y-\alpha \mu^{2} x
\end{gathered}
$$

Therefore, (22) becomes:

$$
\lambda^{3}+A \lambda^{2}+B \lambda+C=0
$$

Differentiating (26) with respect to $\omega$ we have:

$$
\lambda_{\omega}=\frac{-\left[A_{\omega} \lambda^{2}+B_{\omega} \lambda+C_{\omega}\right]}{3 \lambda^{2}+2 A \lambda+B}
$$

Here the index $\omega$ indicates differentiation with respect to $\omega$.

Using Hopf's bifurcation theorem, we set $\omega=0$, $\lambda=i q$ with $q \neq 0$.

$$
\begin{gathered}
\lambda_{\omega}=\frac{-\left\lfloor A_{\omega}(i q)^{2}+B_{\omega}(i q)+C_{\omega}\right\rfloor}{3(i q)^{2}+2 A(i q)+B} \\
\lambda_{\omega}=\frac{A_{\omega} q^{2}-i q B-C_{\omega}}{-3 q^{2}+2 i q A B}
\end{gathered}
$$

Rationalizing (28) we have

$$
\lambda_{\omega}=\frac{\left[\begin{array}{l}
-3 q^{2}\left(A_{\omega} q^{2}-i q B_{\omega}-C_{\omega}\right) \\
-2 i q A\left(A_{\omega} q^{2}-i q B_{\omega}-C_{\omega}\right) \\
+B\left(A_{\omega} q^{2}-i q B_{\omega}-C_{\omega}\right)
\end{array}\right]}{\left[\begin{array}{l}
-3 q^{2}\left(-3 q^{2}+2 i q A+B\right) \\
-2 i q A\left(\begin{array}{l}
-3 q^{2}+2 i q A \\
+B
\end{array}\right)\left(\begin{array}{l}
-3 q^{2} \\
+2 i q A+B
\end{array}\right) \\
+B
\end{array}\right]}
$$

The real part of (29) is given by:

$$
\operatorname{Re} \lambda_{\omega}=\frac{\left(A_{\omega} q^{2}-C_{\omega}\right)\left(B-3 q^{2}\right)-2 q^{2} A B_{\omega}}{\left(B-3 q^{2}\right)^{2}+4 q^{2} A^{2}}
$$

By Akinwade (1996) the sufficient condition for $\operatorname{Re} \lambda_{\omega}>0$ is for the numerator of (30) to be strictly non-negative

i.e.

$$
\begin{gathered}
\left(A_{\omega} q^{2}-C_{\omega}\right)\left(B-3 q^{2}\right)-2 q^{2} A B_{\omega}>0 \\
-3 A_{\omega} q^{4}+\left(A_{\omega} B+3 C_{\omega}-2 A B_{\omega}\right) q^{2}-B C_{\omega}>0
\end{gathered}
$$

Equation (32) is a quadratic in $q^{2}$, thus sufficient conditions for it to hold are that:

$$
-3 \mathrm{~A}_{\omega}>0
$$

And

$$
\left(A_{\omega} B+3 C_{\omega}-2 A B_{\omega}\right)^{2}-12 A_{\omega} B C_{\omega}<0
$$

Assuming that (34) holds. From (33) we have that:

$$
A_{\omega}<0
$$


Recall from (23)

$$
\begin{gathered}
A=-[\alpha(x-y)-(\gamma+\delta+3 \mu)] \\
A_{\omega}=3 \mu_{\omega}-\alpha x_{\omega}+\alpha y_{\omega}
\end{gathered}
$$

Recall from (20)

$$
\begin{array}{r}
\mu=\frac{-(\gamma+\delta)+\sqrt{(\gamma+\delta)^{2}+4 \alpha(\beta+\omega)}}{2} \\
\mu_{\omega}=\frac{\alpha}{\sqrt{(\gamma+\delta)^{2}+4 \alpha(\beta+\omega)}}
\end{array}
$$

Recall from (11) and (12)

$$
\begin{gathered}
x=\frac{\gamma+\delta+\mu}{\alpha} \text { and } y=\frac{\alpha \beta-\mu(\gamma+\delta+\mu)}{\alpha(\gamma+\delta+\mu)} \\
x_{\omega}=\frac{1}{\sqrt{(\gamma+\delta)^{2}+4 \alpha(\beta+\omega)}} \\
\left.y_{\omega}=\frac{-2 \alpha \beta}{\left[\left[\begin{array}{l}
(\gamma+\delta)^{2}+2 \alpha(\beta+\omega) \\
+(\gamma+\delta) \sqrt{(\gamma+\delta)^{2}} \\
+4 \alpha(\beta+\omega)
\end{array}\right] \sqrt{(\gamma+\delta)^{2}}+4 \alpha(\beta+\omega)\right.}\right] \\
-\frac{\left[\begin{array}{l}
1 \\
\sqrt{(\gamma+\delta)^{2}+4 \alpha(\beta+\omega)}
\end{array}\right.}{}
\end{gathered}
$$

Substituting (37), (38) and (39) into (36) we have

$$
\begin{aligned}
& A_{\omega}=\frac{2 \alpha}{\sqrt{(\gamma+\delta)^{2}+4 \alpha(\beta+\omega)}} \\
& -\frac{2 \alpha^{2} \beta}{\left[\begin{array}{l}
(\gamma+\delta)^{2}+2 \alpha(\beta+\omega) \\
+(\gamma+\delta) \sqrt{\begin{array}{l}
(\gamma+\delta)^{2} \\
+4 \alpha(\beta+\omega)
\end{array}}
\end{array}\right] \sqrt{\left.\begin{array}{l}
(\gamma+\delta)^{2} \\
+4 \alpha(\beta+\omega)
\end{array}\right]}}
\end{aligned}
$$

At $\omega=0$

$$
A_{\omega}=\frac{\left[\begin{array}{l}
2 \alpha\left[\begin{array}{l}
(\gamma+\delta)^{2}+2 \alpha \beta \\
+(\gamma+\delta) \sqrt{(\gamma+\delta)^{2}} \\
+4 \alpha \beta
\end{array}\right]-2 \alpha^{2} \beta
\end{array}\right]}{\left[\begin{array}{l}
(\gamma+\delta)^{2}+2 \alpha \beta \\
+(\gamma+\delta) \sqrt{(\gamma+\delta)^{2}} \\
+4 \alpha \beta
\end{array}\right] \sqrt{(\gamma+\delta)^{2}+4 \alpha \beta}}
$$

Using (35) on (41) gives

$$
(\gamma+\delta)^{2}+\alpha \beta+(\gamma+\delta) \sqrt{(\gamma+\delta)^{2}+4 \alpha \beta}<0
$$

Let

$$
J_{2}=(\gamma+\delta)^{2}+\alpha \beta+(\gamma+\delta) \sqrt{(\gamma+\delta)^{2}+4 \alpha \beta}
$$

Clearly from (43), $J_{2}>0$ which imply that the Endemic Equilibrium (EE) state does not bifurcate, i.e. indicating that the state is stable.

The implication of this is that the Measles may become prevalent in the population but may not be endemic.

The table below justifies our analysis.

\section{Results and Discussion}

Table 1. The Bifurcation Analysis of Endemic Equilibrium State

\begin{tabular}{|c|c|c|c|c|c|}
\hline$\alpha$ & $\beta$ & $\delta$ & $\gamma$ & $\mathrm{J}_{2}$ & REMARK \\
\hline 0.001 & 0.2 & 0.01 & 0.15 & 0.051797 & STABLE \\
\hline 0.002 & 0.2 & 0.01 & 0.15 & 0.052388 & STABLE \\
\hline 0.003 & 0.2 & 0.01 & 0.15 & 0.052973 & STABLE \\
\hline 0.004 & 0.2 & 0.01 & 0.15 & 0.053553 & STABLE \\
\hline 0.005 & 0.2 & 0.01 & 0.15 & 0.054127 & STABLE \\
\hline 0.006 & 0.2 & 0.01 & 0.15 & 0.054697 & STABLE \\
\hline 0.007 & 0.2 & 0.01 & 0.15 & 0.055262 & STABLE \\
\hline 0.008 & 0.2 & 0.01 & 0.15 & 0.055822 & STABLE \\
\hline 0.009 & 0.2 & 0.01 & 0.15 & 0.056377 & STABLE \\
\hline 0.01 & 0.2 & 0.01 & 0.15 & 0.056928 & STABLE \\
\hline
\end{tabular}




\section{Conclusion}

The Disease Free equilibrium state will be stable if $\frac{\alpha \beta}{\mu}<(\gamma+\delta+\mu)$ that is the population is sustainable. We went further to define a bifurcation parameter $\omega$ from the condition of stability of zero equilibrium state and use it to analyze the non-zero equilibrium state. The $\omega$ transit from positives to negative, when it is negative it implies disease outbreak otherwise no outbreak. The result was tested by hypothetical values and it shows no indication of outbreak of disease epidemics, which implies that once the disease enters into a population, it is likely to persist.

\section{Acknowledgements}

My sincere gratitude goes to Prof. N. I. Akinwande and Prof. K. R. Adeboye that took time out of their tight schedule to guide us and look through this work over and over again.

\section{REFERENCES}

[1] Abubakar, S., Akinwande, N. I. \& Abdulrahman, S. (2012). A Mathematical Model of Measles Disease Dynamics. Journal of Science, Technology, Mathematics and Education (JOSTMED) 8(3), 117-122.

[2] Akinwande, N. I. (1996). A Mathematical Model of Yellow Fever Epidemics. Afrika Mathematika, 6: 56-58.

[3] Grimshaw, R. (1990). Nonlinear Ordinary Differential Equations. Black Scientific Publications, Boston Melbourne. Pp 245.

[4] Bjørnstad, O.N., Finkenstädt, B., and Grenfell, B.T. (2002) Dynamics of Measles Epidemics: Estimating Scaling of Transmission Rates using a Time Series SIR Model Ecological Monographs 72 169-184. 